\title{
An efficient and accurate method to calculate diffusion coefficient of structured particles. A first case study of $\mathrm{Pb}$ diffusion in rare gases
}

\author{
Ján Matúška \\ Institute of Physical Chemistry and Chemical Physics, FCHPT-STU, \\ Radlinského 9, 812 37, Bratislava, Slovakia \\ jan.matuska@stuba.sk
}

\begin{abstract}
Diffusion coefficient depends on temperature, pressure, reduced mass of colliding particles and collision cross section. The presented method is designed to calculate the diffusion coefficient in loose systems containing molecules with relatively complicated colliding trajectories. It is a combination of the ChapmanEnskog theory and the molecular dynamics calculation. The Chapman-Enskog theory provides the relation between the diffusion coefficient and the collision cross section which is the result of multiple integration of the scattering angle of all possible initial conditions of the collision. The scattering angle is obtained by numerical integration of the Newton's equation of motion with previously selected initial conditions. The proposed method has been verified for the simple system of a lead atom diffusion in rare gases and the results were compared to those of two other theoretical methods.
\end{abstract}

Keywords: diffusion coefficient, molecular dynamics, rare gas, heavy atoms

\section{Introduction}

Rare-gas atoms (RG) are frequently used in experiments involving transport properties as a carrier gas (Koperski 2002). The studied particles range from light (Soldán et al., 2001) to heavy (Lee et al., 2007; Gardner et al., 2010) elements and from single atomic species to macromolecules (Mesleh et al., 1996). The transport coefficient heavily depends on the interaction potential of the diffusing element and the carrier gas as well as on the macroscopic conditions. Correct and accurate description of the interaction potential is possible by ab-initio methods; however, the relation between the interaction potential and the resulting transport coefficient is not a straightforward one. There are multiple methods to calculate transport coefficient from a known potential-energy curve. The velocity autocorrelation function (VACF) can be used effectively to evaluate the transport properties upon a molecular dynamics (MD) simulation (Hoheisel and Vogelsang, 1988). However, this approach is effective only in liquids and solids; its convergence in gases is slow. A more effective way is employing the scattering theory (SC) which relies on the evaluation of collision integrals and subsequent solution of the Boltzmann equation. Here, the crucial part is the evaluation of the collision integral using various methods. In case of structureless particles (atoms), the method of Viehland and Chang (Viehland and Chang, 2010), based on the numerical integration of the analytical expression for the scattering angle, can be accurately applied.
This method is unusable in case of structured particles because the analytical expression for the scattering angle is generally not determined. Therefore, a method based on the numerical integration of motion is a much more viable option (Mesleh et al., 1996) considering structured particles.

The main aim of this work is the proposition, implementation and verification of a method based on the scattering theory and molecular dynamics. Verification was performed for a system of a lead atom diffused in an RG carrier gas. The diffusion coefficient was calculated and compared to experimental values and those obtained by other theoretical methods at various temperatures.

\section{Methods}

\section{Theoretical background}

The presented method is designed to calculate the diffusion coefficient in gases. In this case, the Chapman-Enskog theory provided the relation between the diffusion coefficient and the collision cross section (Mason and McDaniel, 1988):

$$
D_{12}=\frac{1}{p} \frac{3}{8} \sqrt{\frac{\pi(k T)^{3}}{2 m_{r}}} \frac{1}{\Omega_{12}(T)}
$$

where $T$ is the temperature, $p$ is the pressure, $k$ is the Boltzmann constant, $m_{r}$ is the reduced mass of the interacting particles and $\Omega_{12}(T)$ is the averaged collision cross section for the collision of a ion and a gas molecule. 
According to Eq. (1), the crucial part of the calculation is the evaluation of the collision cross section which is calculated by averaging the scattering angle of the initial conditions related to the desired temperature $T$. In case of atom $\mathrm{Pb}$ and an RG atom, the integral has the form:

$\Omega_{12}(T)=$
$=\int_{v_{r}=0}^{\infty} \int_{b=0}^{\infty} \frac{\pi}{8}\left(\frac{m_{r}}{k T}\right)^{3} v_{r}^{5} e^{-\frac{m_{r} v_{r}^{2}}{2 k T}} 2 b\left(1-\cos \left(\kappa\left(v_{r}, b\right)\right)\right) d b d v_{r}$

where $b$ is the impact parameter, $v_{r}$ is the relative velocity and $\kappa$ is the scattering angle.

Obtaining the analytic expression of the scattering angle dependence on the initial conditions (relative velocity, impact parameter) is a nontrivial task fully accomplished only for the simplest cases. Even then, the evaluation of the multiple integral in Eq. (2) is a nontrivial problem that can be solved by the Monte-Carlo method. Initial conditions are generated with respect to the desired temperature and distribution function. However, the maximum impact parameter has to be limited to some finite value, which can result in some uncertainty in the calculation proportionally to the strength of the neglected interaction beyond the maximum value of the impact parameter. Therefore, such an uncertainty is negligible for large values of the maximum impact parameter. Then, the trajectory of each initial condition is calculated by numerical integration of the Newton's equation of motion with respect to the interaction potential. The ob- tained velocities of the interacting particles were used to calculate the value of the scattering angle. The Monte Carlo integration is finished by averaging the scattering angle applying the integral in Eq. (2).

\section{Computational details}

A scheme of the calculation is presented in Fig. 1. The interaction potential (using a cubic $\mathrm{C}^{1}$ continuous b-spline interpolation) was based on the extrapolated X2C/CCSD(T) Pb...RG potential curves presented by Sladek et al. (2014). The cubic $\mathrm{C}^{1}$ continuous b-spline interpolation ensures a continuous first derivative of the potential curve in the molecular dynamic calculation (Berendsen, 2007). Generation of the initial condition and numerical integration of motion were performed in the computational code ANT 13 (Li, 2013). The initial condition was generated with respect to the desired temperature (1100-1300 K) and maximum impact parameter, which was determined by the potential cutoff. The exact value is showed in Tab. 1 and it depends on the quality and properties of the source potential. A set of 1250000 initial conditions was created. The trajectory calculation was carried out at constant energy using the velocity Verlet integrator. Time-step of the numerical integration was $0.5 \mathrm{fs}$.

The output of ANT 13 is the final positions and momentums after the collision. A further evaluation of these quantities had to be done using a custom code written in Python 2.7 to obtain the scattering angle and the collision cross section.

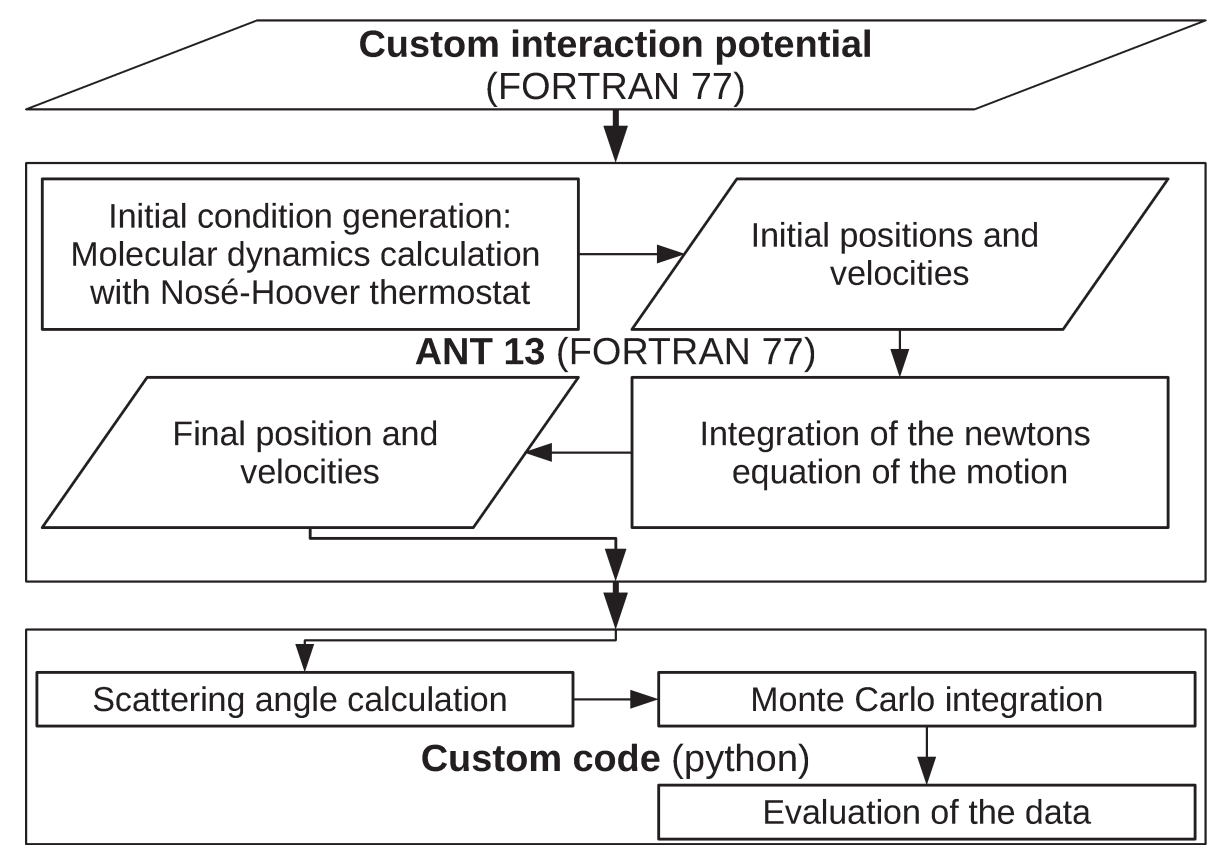

Fig. 1. Calculation scheme; detailed description of the parameters and procedures is provided in Computational details. 
Tab. 1. Potential cutoff $\left(r_{\text {cut }}\right)$ for the studied systems determined by the maximal value of the calculated X2C/CCSD(T) interaction potential (Sladek et al., 2014).

\begin{tabular}{lc}
\hline system & $r_{\text {cut }} / \AA$ \\
\hline $\mathrm{Pb} . . \mathrm{Ar}$ & 20 \\
$\mathrm{~Pb} . . \mathrm{Ne}$ & 17 \\
$\mathrm{~Pb} . . \mathrm{He}$ & 20 \\
\hline
\end{tabular}

\section{Results}

The herein considered parameter is the pressureindependent cross-diffusion coefficient $p D$ of the mutual diffusion of the $\mathrm{Pb}$ atom in $\mathrm{RG}$ as it allows an easy comparison of a wide range of experimental and theoretical results. The experimental and theoretical results were compared for temperatures: $1100 \mathrm{~K}, 1150 \mathrm{~K}, 1200 \mathrm{~K}, 1250 \mathrm{~K}, 1300 \mathrm{~K}$.

Experimental data were taken from the work of Arefiev et al. (1974) and theoretical data originate from Sladek et al. (2014). Sladek et al. calculated the diffusion coefficient using tree independent approaches. Results of two of them were compared to those obtained in our work.

The first theoretical reference approachis based on the evaluation of the diffusion coefficient by means of the scattering theory as is implemented in the VARY and PC codes (Viehland and Chang, 2010; Viehland et al., 1995). This method is considered to be the most accurate one.

The second theoretical reference approach employs molecular dynamics simulation of the studied species. Time evolution of the $\mathrm{Pb}$ velocity is used to obtain the velocity autocorrelation function (VACF) of the $\mathrm{Pb}$ atom in an RG gas and the integral of VACF is proportional to the diffusion coefficient.

The presented method of the diffusion coefficient evaluation is a combination of these two methods. Calculation of the diffusion coefficient by the equation (1) is the simplest possible version of the scattering theory calculation and the collision cross section calculation is based on the molecular dynamic calculation of the colliding pairs of atoms.

Tab. 2. Computational cost of molecular dynamics calculation and of the presented method at the temperature $1100 \mathrm{~K}$.

\begin{tabular}{lcc}
\hline system & $\begin{array}{c}\text { MD calculation } \\
\text { hours }\end{array}$ & $\begin{array}{c}\text { Presented method } \\
\text { hours }\end{array}$ \\
\hline $\mathrm{Pb} . . \mathrm{Ar}$ & $4389: 30$ & $01: 50$ \\
$\mathrm{~Pb} . \mathrm{Ne}$ & $4213: 25$ & $01: 12$ \\
$\mathrm{~Pb} . \mathrm{He}$ & $4213: 00$ & $00: 42$ \\
\hline
\end{tabular}

\section{Pb..Ar}

Results for $\mathrm{Pb}$..Ar are shown in Fig. 2 and they are in excellent agreement with the calculation of the diffusion coefficient by the VARY and PC codes which is to be taken as the theoretical limit. The molecular dynamics results (obtained by the integration of VACF) are also in agreement with the experiment but their error margins are bigger.

\section{Pb..Ne}

A comparison of the predicted diffusion coefficient is provided in Fig. 3. The agreement between the presented results and the results obtained using the VARY and PC codes is similar as in the previous system. However, molecular dynamics results severely underestimate the diffusion coefficient and their uncertainty is higher than the uncertainty observed for other theoretical methods.

\section{Pb..He}

Fig. 4 presents the results obtained for the $\mathrm{Pb}$..He system. The agreement between the presented results and those of the VARY and PC codes is within the error margins of the presented method for every temperature studied. Both mentioned results are also in agreement with the experimental ones (considering the experimental uncertainty). Molecular dynamics results underestimate the other two theoretical results. The value of $p D_{12}$ obtained by molecular dynamics simulations is underestimated by about $15 \%$. Moreover, the molecular dynamics results failed to reliably reproduce the expected trend of the dependence and the experimental results.

The results of the proposed method are in agreement with the more accurate scattering method implemented in the PC and VARY codes for the whole range of carrier gases and temperatures. On the contrary, deviations between the molecular dynamics method and the other two theoretical approaches increase with the decreasing mass of the RG atom.

An analysis of the computational cost showed that the proposed calculation is faster than the molecular dynamics simulation (which takes several days), see Table 2. The main problem of the molecular dynamics simulation in gases is the slow convergence of VACF due to the rare collisions and weak interaction between the studied atoms, therefore the calculation time is long and even then the uncertainty of the results is high. However, the proposed method is slower than the evaluation of the collision integral by the VARY and PG codes (minutes on a personal computer). This disadvantage is compensated by the calculation not being limited to a certain type of numerically integrable inter- 


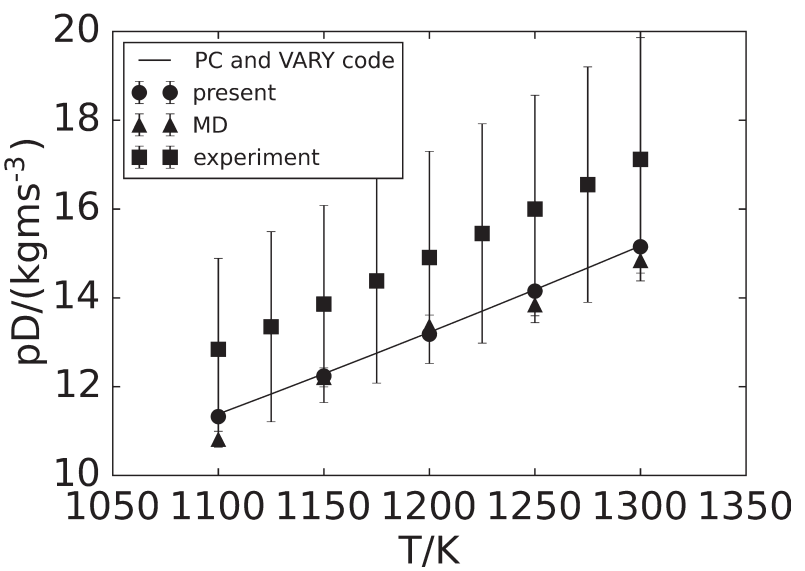

Fig. 2. Temperature dependence of $p D_{12}$ for $\mathrm{Pb}$ diffusion in Ar in the temperature range of 1100-1300 K calculated by different approaches and compared to experimental results. The experimental (Arefiev et al., 1974) values for $\mathrm{Pb}$ in $\mathrm{Ar}$ are depicted with a $16 \%$ relative error bar. The extrapolated dU[DT]Z X2C/CCSD(T) curves (Sladek et al., 2014) for Pb..Ar were used to calculate of $p D_{12}$.

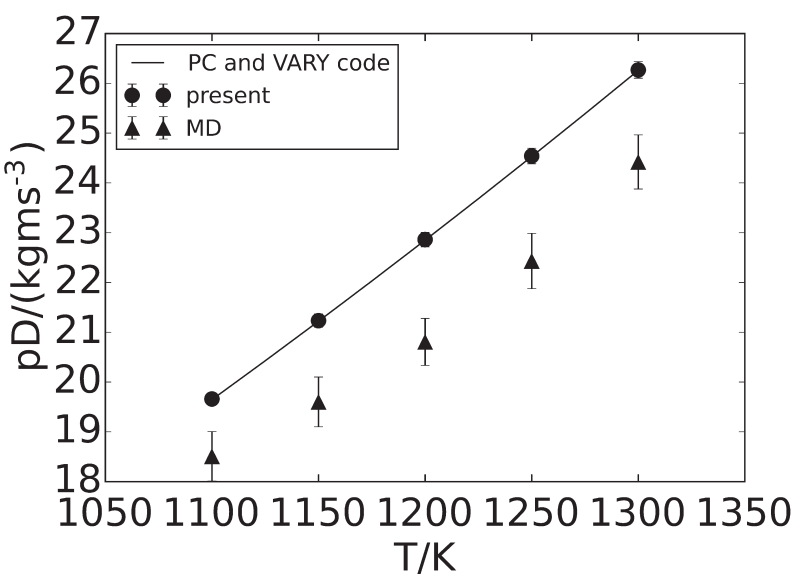

Fig. 3. Temperature dependence of $p D_{12}$ for $\mathrm{Pb}$ diffusion in $\mathrm{Ne}$ in the temperature range of 1100-1300 K. The extrapolated dU[DT]Z X2C/ CCSD(T) curves (Sladek et al., 2014) for Pb..Ne were used to calculate $p D_{12}$.

action potentials. In principle, any force field parametrization for a given structure of interacting particles (molecules) can be used.

\section{Conclusion}

Results of the presented method were compared to those of two other calculation methods and to those obtained experimentally. This comparison has confirmed that the presented approach provides reliable results of the diffusion coefficient in the gas phase. The difference between the presented method and

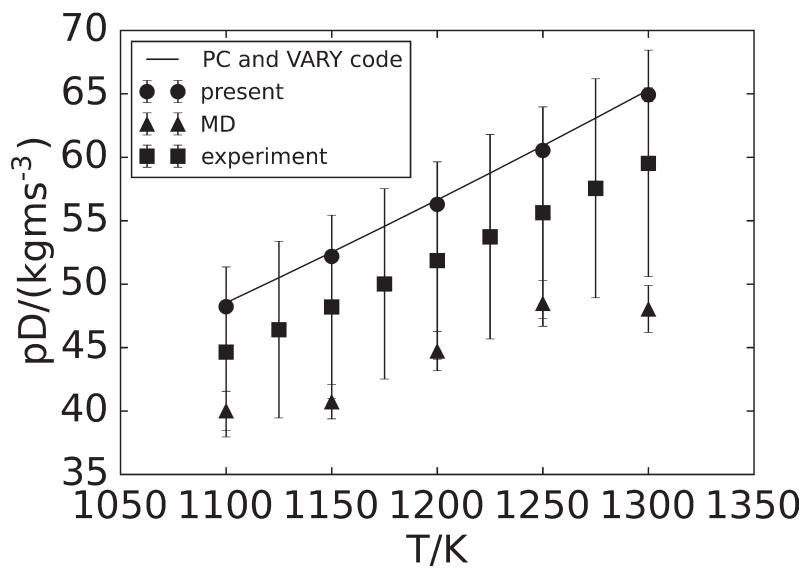

Fig. 4. Temperature dependence of $p D_{12}$ for $\mathrm{Pb}$ diffusion in He in the temperature range of 1100-1300 K. The experimental (Arefiev et al., 1974) values for $\mathrm{Pb}$ diffusion in $\mathrm{He}$ are depicted with a $15 \%$ relative error bar. The extrapolated dU[TQ]Z curves (Sladek et al., 2014) for Pb..He were used to calculate $p D_{12}$.

the more accurate scattering theory implemented in the VARY and PC codes is within the error margins for every considered calculation (temperature) and/ or system. The computational cost is higher but the presented method is not limited to the interaction potential of atomic species as is the case of the scattering theory implemented in the VARY and PC codes. The presented method is much more accurate than the simple molecular dynamics calculation approach and the computation cost is much more in favor of the presented method.

\section{Acknowledgement}

This work was supported by the Slovak Grant Agency (VEGA 1/0327/12). We are also grateful to the HPC Center at the Slovak University of Technology in Bratislava, which is a part of the Slovak Infrastructure of High Performance Computing (SIVVP project, ITMS code 26230120002, funded by the European Regional Development Fund, ERDF), for the computational time and resources provided.

\section{References}

Arefiev KM, Lesjuis AR, Zamchenkov BM and Chemezman LSch, (1974) J. Eng. Phys. 27: 825.

Berendsen HJC (2007) Simulating the Physical World: From Quantum Mechanics to Fluid Dynamics, Cambridge University Press, Cambridge.

Gardner AM, Withers CD, Wright TG, Kaplan KI, Chapman CYN, Viehland LA, Lee EPF and Breckenridge WH (2010) J. Chem. Phys., 132, 054302.

Hoheisel C and Vogelsang R (1988) Computer Physics reports 8: 1-69. 
Koperski J (2002) Phys. Rep. 369: 177.

Lee EPF, Gray BR, Joyner NA, Johnson SH, Viehland LA, Breckenridge WH and Wright TG (2007) Chem. Phys. Lett. 450: 19.

Li Z-H, Jasper AW, Bonhommeau DA, Valero R, Zheng J and Truhlar DG (2013) ANT 2013, University of Minnesota, Minneapolis.

Mason EA, McDaniel EW (1988) Transport Properties of Ions in Gases, Wiley, New York.

Mesleh MF, Hunter JM, Shvartsburg AA, Schatz GC and Jarrold MF (1996) The Journal of Physical Chemistry 100: 16082-16086.
Sladek V, Bucinsky L, Matuska J, Ilcin M, Lukes V and Laurinc V (2014) Phys. Chem. Chem. Phys. 16: 18519-18532.

Soldán P, Lee EPF, Lozeille J, Murrell J N and Wright TG (2001) Chemical Physics Letters 343: 429-436.

Viehland LA and Chang Y (2010) Computer Physics Communications 181: 1687-1696.

Viehland LA, Janzen AR and Aziz RA (1995) J. Chem. Phys., 102: 5444. 